\title{
Modelización sostenible del emplazamiento de nuevas segundas residencias en el medio rural. El caso de La Vera (Extremadura)
}

\section{Sustainable modeling of new second homes placement in rural area. The case of La Vera (Extremadura)}

\author{
Jin Su Jeong ${ }^{1}$, Lorenzo García Moruno ${ }^{2}$, Julio Hernández Blanco ${ }^{3}$ y \\ Francisco Javier Jaraíz Cabanillas ${ }^{4}$
}

\section{INTRODUCCIÓN}

En la actualidad, el ecoturismo es un tema de investigación recurrente que ha sido identificado o definido como una forma sostenible de turismo, que se espera que contribuya tanto a la conservación ambiental como al desarrollo económico del espacio en el que se da (Ross \& Wall, 1999; Vincent \& Thompson, 2002). Por consiguiente, el ecoturismo se reconoce como un modo sostenible para desarrollar regiones con abundantes recursos turísticos (Weaver, 2001; Weaver \& Lawton, 2007). Sin embargo, debido a las insufi-

\footnotetext{
${ }^{1}$ Investigador Postdoctoral en el Departamento de Expresión Gráfica del Centro Universitario de Mérida de la Universidad de Extremadura. (jin@unex.es). Jin Su Jeong agradece la financiación de la Universidad de Extremadura (Contrato puente para becarios predoctorales).

2 Profesor Titular en el Departamento de Expresión Gráfica del Centro Universitario de Mérida de la Universidad de Extremadura (lgmoruno@unex.es).

${ }^{3}$ Profesor Titular en el Departamento de Expresión Gráfica del Centro Universitario de Plasencia de la Universidad de Extremadura (juliohb@unex.es).

${ }^{4}$ Profesor Sustituto en el Departamento de Ciencias del Territorio de la Universidad de Extremadura (jfjaraiz@unex.es).
} 
cientes evaluaciones ambientales y las actuaciones llevadas a cabo con anterioridad en estos territorios, muchos destinos turísticos se están poniendo en peligro o pueden considerarse como autodestructivos para sus entornos. En el sector turístico, el desarrollo sostenible ha sido ampliamente considerado porque cada proceso o avance puede atender a las demandas de los turistas, proteger localizaciones físicas y proporcionar oportunidades para mejorar el crecimiento económico y la calidad de vida de los residentes, a través de la coexistencia del desarrollo turístico y la calidad ambiental (Eagles et al., 2002; Morales Yago, 2013). Con el crecimiento del interés ecológico y del patrimonio cultural, las mejoras en la calidad ambiental local aumentan la atracción visual y también realzan los valores estéticos y recreativos de estos ambientes (Zhang \& Lei, 2012). Consecuentemente, los residentes locales obtienen beneficios de su medio ambiente local mejorado, lo que provoca a su vez una mayor calidad de vida (Allen et al., 1993). Por otro lado, la opinión popular en relación a las segundas viviendas está firmemente enraizada en el medio rural, pues esta está considerada como el emplazamiento óptimo de las segundas residencias, así como el medio o el espacio en el que desarrollar las actividades que permitan ganarse la vida a los residentes (Gallent $\&$ Tewdwr-Jones, 2000; Tewdwr-Jones et al., 2002; Vepsäläinen \& Pitkänen, 2010). La actual planificación u ordenación territorial regional, no se ha desarrollado o modificado convenientemente para tratar los nuevos cambios del medio rural, que afectan a la localización de nuevas casas de campo, las cuales siguiendo y cumpliendo ciertos criterios podrían mitigar sus impactos negativos en el medio rural (Bell, 1995; García Moruno et al., 1998; Tandy, 1979).

Desde el modelo actual de desarrollo local, el énfasis que se hace en las segundas residencias de la comunidad local, se debe al deseo de conservar los recursos locales e incrementar los beneficios en estos espacios. Con esto se alude a la cercana asociación entre el ecoturismo y los residentes locales, que son uno de los principales o esenciales grupos de inversores en estas viviendas (Byrd et al., 2009). La postura de los residentes ha sido examinada de cerca para intentar comprender el nivel de apoyo de estos hacia el turismo, así como su relación con el impacto percibido desde el turismo (Andereck et al., 2005; Teye et al., 2002). Teniendo en cuenta la actitud de los residentes hacia el ecoturismo, es un prerrequisito incorporar su participación en estos estudios (Page \& Dowling, 2002). Comprender la postura de los residentes hacia los principios de gestión del ecoturismo, puede ayudar a quienes toman las decisiones a concebir estrategias de gestión más eficientes y apropiadas, y arreglar posibles conflictos entre la conservación de los recursos locales y el desarrollo económico (Lai \& Nepal, 2006). Así reflexionando sobre estos hechos y anali- 
zando sus implicaciones, quienes toman las decisiones en materia urbanística y de ordenación del territorio, pueden encontrar respuesta al estado actual de la cuestión y recibir ideas para mejorar las condiciones de futuro (Jeong et al., 2014a). Lo ideal es que las posibles consecuencias de los planes y políticas bajo consideración y la participación de los residentes, contribuyan a distinguir la calidad en la gestión del ecoturismo (Blaschke, 2006; Drumm, 1998). A la hora de estudiar la modelización espacial, los sistemas de información geográfica (en adelante SIG) son una herramienta particularmente útil para analizar grandes volúmenes de datos espaciales, relacionados con las políticas económicas, sociales, culturales y ecológicas (Böhme \& Schön, 2006; Hermann \& Osinski, 1999). Los procesos jerárquicos analíticos (en adelante AHP, de sus siglas en inglés) incorporados a la tecnología SIG, producen un modo flexible de combinar varios criterios en el proceso de localización (Saaty, 1977). La toma de decisión multi-atributo (en adelante MADM, de sus siglas en inglés) es un tipo particular de ordenación o planificación espacial para apoyar u orientar a los decisores políticos y empresariales a la hora de explorar y solventar sus múltiples y complicados problemas (Malczewski, 1999; Roy, 1996). Solo algunos estudios se han centrado en la materia presentada, incluyendo la evaluación de varios criterios y la agregación de estos criterios en diferentes procesos (De Vriesa et al., 2012; Jeong et al., 2012; Jeong et al., 2014b; Tassinari \& Torreggiani, 2006).

El presente trabajo describe un método de ordenación espacial para determinar los emplazamientos más adecuados para segundas nuevas residencias, basado en la comprensión de las limitaciones de la ordenación territorial regional existente en el norte de la región de Extremadura, concretamente en la comarca natural de La Vera. La metodología propuesta usa las funciones del AHP y el promedio ponderado ordenado (en adelante OWA, de sus siglas en inglés) con un valor constante de orness y una máxima entropía de modelado multi-atributo. Con el fin de evaluar por completo el área de estudio, se usa una clasificación común de escala en un entorno SIG (Eastman, 2003; Fuller \& Majlender, 2001; Yager, 1988). El objetivo de la investigación es mostrar un nuevo enfoque empírico que permita integrar nuevas segundas residencias rurales mediante el análisis de los ecosistemas existentes, las infraestructuras y el medio físico, a través de los métodos que se proponen en este trabajo. La selección de criterios se ha limitado a los datos básicos que están actualmente disponibles y que proceden de diferentes fuentes, por consiguiente, se reconoce que se han omitido algunos de los factores claves que potencialmente podrían producir diferentes alternativas de decisión. Adicionalmente, se propone dar explicación a las diferentes decisiones alternativas y/o patrones, que 
permitan evaluar un método razonable de sostenibilidad del ecoturismo y su integración. En consecuencia, la intención de implicar a la población local se justifica a través de los resultados, siendo muy instructivo a la hora de demostrar la actitud participativa de los residentes en el planeamiento u ordenamiento de los territorios para fomentar el ecoturismo.

\section{MATERIALES Y MÉTODO}

El área de estudio, la comarca natural de La Vera $\left(888 \mathrm{~km}^{2}\right)$, se encuentra en la provincia de Cáceres, concretamente en la vertiente sur de la Sierra de Gredos en el noreste de la región de Extremadura (figura 1). Esta región está limitada por el Valle del Jerte en el noroeste (Extremadura), la región de Castilla y León en el norte, la provincia de Toledo en el este y la comarca natural del Campo Arañuelo y el río Tiétar en el sur (Extremadura). La comarca de La Vera está formada por 19 municipios. Su proximidad a Madrid y las condiciones naturales (paisajes naturales y recursos hídricos) han provocado que la comarca se haya convertido en un importante reclamo turístico y en un espacio en el que han aumentado de un modo considerable las residencias de fin de semana de la población capitalina.

Por consiguiente, las segundas viviendas de campo son una parte integrante del paisaje recreativo rural tanto histórico como contemporáneo (Gallent \& Tewdwr-Jones, 2000; Vepsäläinen \& Pitkänen, 2010). Este crecimiento ha provocado cambios importantes en los patrones de usos del suelo, incrementado el porcentaje de población diseminada, con sus consecuentes impactos. Durante las últimas décadas, el desarrollo de construcciones rurales, motivado por el crecimiento de las residencias de vacacionales, y los entornos naturales han impulsado a su vez las actividades turísticas, como muestra el significativo incremento de la construcción de nuevos hoteles y casas rurales (Jaraíz et al., 2013). Especialmente, esto se refleja también en las tendencias que muestran los datos de viviendas junto a los de población, en los que se puede apreciar (figura 2) que el número total de segundas residencias ha aumentado mientras que el número de viviendas habituales o primeras residencias ha disminuido y las vacías han crecido (INE, 2011).

Puesto que el trabajo se refiere a la construcción de viviendas integradas en el entorno rural, es necesario comenzar definiendo los conceptos geográficos relacionados con el poblamiento. El término construcción residencial, denota premisas de una o varias habitaciones además de cuartos auxiliares, y que haya sido especialmente construida para cumplir las funciones de vida resi- 


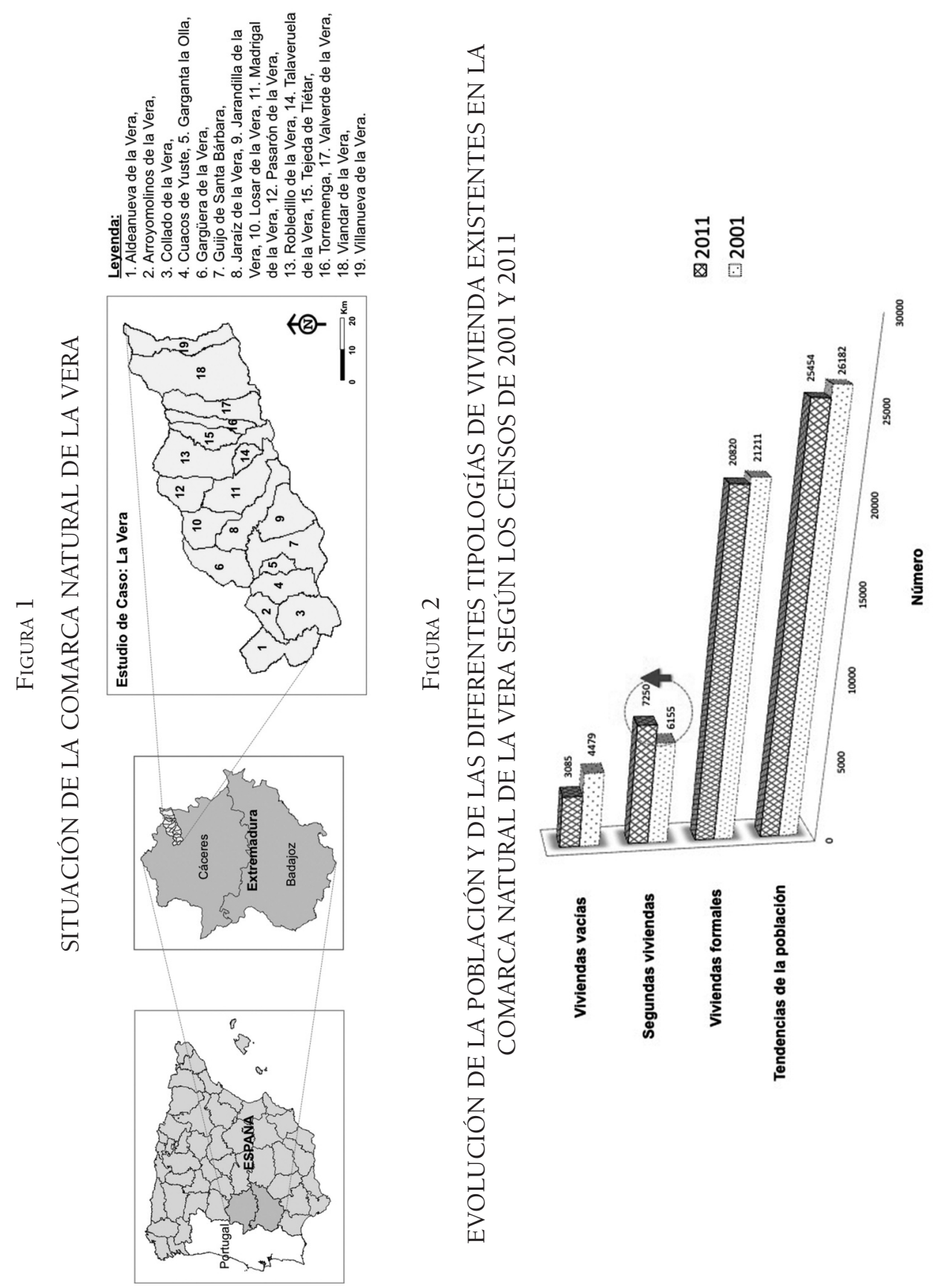

Estudios Geográficos, Vol. LXXVII, 280, pp. 191-216, enero-junio 2016 ISSN: 0014-1496, eISSN: 1988-8546, doi: 10.3989/estgeogr.201607 
dencial. En el área de estudio propuesta, debe enfatizarse que esta construcción residencial, se trata de una vivienda unifamiliar en las que el número de las mismas se encuentran en función del tamaño del centro urbano más próximo y de la distancia a él de toda su área de influencia (alrededor del 30\% de las segundas residencias en total).

\section{Metodología de la investigación}

Para identificar la ubicación más apropiada para la construcción de segundas viviendas rurales en el área de estudio, se propone un proceso de evaluación multi-criterio considerando varios conjuntos de criterios (figura 3). Estos conjuntos formaron una estructura jerárquica multi-criterio para evaluar una planificación sostenible. Los criterios de evaluación se determinaron por los autores basándose en legislación europea en cuanto a la edificación de viviendas en ambientes rurales (Council of the European Union, 2001), la Ley del Suelo y Ordenación del Territorio de Extremadura (LESOTEX, 2001) y a través de una revisión bibliográfica de la litera más relevante relacionada con los criterios de evaluación utilizados (Carver, 1999; Jeong et al., 2013; 2015).

Aunque los pesos de los criterios se basan objetivamente en datos reales y se expresan en una escala de medida, la asignación de pesos en el proceso del MADM se considera parcialmente subjetiva porque depende de las decisiones tomadas por los autores. Para lograr criterios consensuados de ponderación y hacerlos más objetivos, se siguió un procedimiento analítico, consistente tanto en una encuesta a los residentes de los diferentes municipios, como la conformación de un grupo de discusión con un panel de expertos en la materia. La encuesta domiciliaria a los residentes locales fue llevada a cabo para conocer sus preferencias en cuanto a los criterios de ponderación. A través de esta encuesta, los participantes asignaban mediante una escala de calificación continua de 9 puntos, sus preferencias en relación a los diferentes criterios de ponderación, a través de una comparación de pares (en adelante PCM, de sus siglas en inglés).

Se obtuvieron un total de 41 respuestas útiles de una población efectiva de 54 residentes. Entre estas respuestas, 23 corresponden a propietarios de segundas viviendas. En el caso de la reunión de expertos, que incluyó profesores, encargados de formular políticas regionales, planificadores y autoridades locales, todos ellos responsables en cierta medida de proyectos de ordenación territorial regional, también se consideró su opinión en el proceso de ponderación de la aplicación, al igual que se hizo con los resultados de la encuesta a los residentes locales, tal y como se acaba de comentar. La técnica OWA, una clase de operadores multi-criterio, fue utilizada para criterios de clasificación y para 
FIGURA 3

\section{MODELIZACIÓN DEL EMPLAZAMIENTO DE NUEVAS SEGUNDAS RESIDENCIAS EN EL MEDIO RURAL}

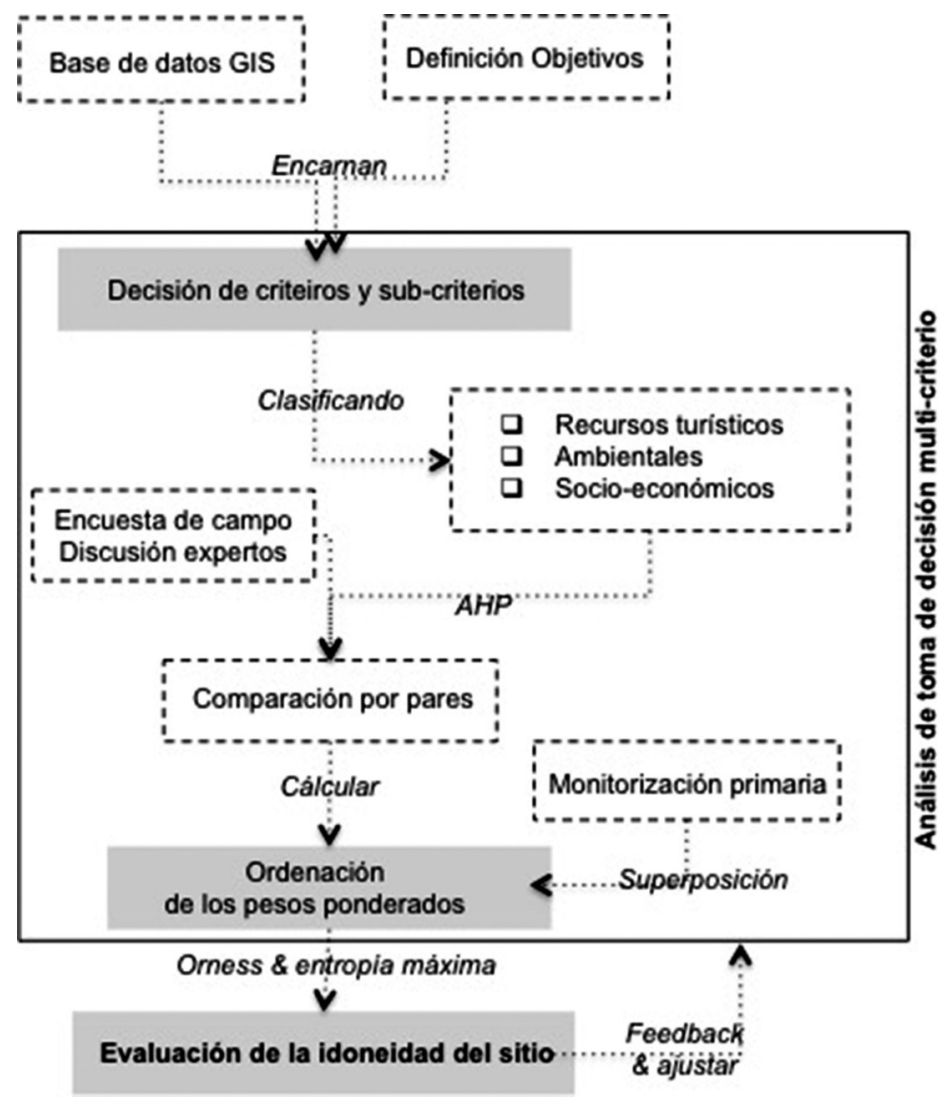

abordar la incertidumbre de sus interacciones (Yager, 1988). En consecuencia, la agregación de los pesos de los criterios y los valores de los atributos en el OWA, puede producir puntuaciones idóneas de las áreas de estudio y puede representar las zonas adecuadas para resolver el problema de los criterios múltiples (Malczewski, 2004). La metodología presentada, inicialmente excluye las áreas inapropiadas, lo cual limita el análisis a áreas geográficas concretas. La metodología, por consiguiente, resultó ser como una evaluación del suelo basada en los índices de idoneidad tras aplicar las restricciones iniciales. 
Evaluación de los criterios de idoneidad del emplazamiento

Un proceso de emplazamiento de segundas viviendas o residencias rurales requiere la consideración de múltiples criterios y niveles de evaluación, para identificar las mejores localizaciones disponibles y eliminar los impactos posteriores y los efectos adversos a largo plazo. Los criterios de evaluación fueron clasificados en cuatro categorías principales, a saber: 1) limitaciones (criterios de exclusión), 2) recursos turístico, 3) criterios ambientales y 4) criterios socio-económicos (tablas 1 a 4). Especialmente, para evaluar la postura de los residentes a la hora de encontrar criterios adecuados de ponderación en la planificación de segundas residencias, los participantes fueron entrevistados para expresar su opinión y evaluar su intención de colaborar en diferentes niveles del proceso de toma de decisiones. La participación de los residentes puede acontecer en diferentes niveles, contribuyendo a la planificación y gestión del ecoturismo y participando en la toma de decisiones dentro del comité de gestión (Guevara, 1996).

En el proceso de cálculo estuvieron involucrados dieciocho sub-criterios, divididos en cuatro grupos principales en función de los pesos calculados (tablas 1 a 4). El primer grupo incluye criterios de restricción que limitan el análisis a áreas geográficas particulares. El segundo grupo se compone de criterios relevantes para los parámetros de los recursos turísticos. El tercer grupo se relaciona con los criterios principales para los parámetros ambientales, mientras que el cuarto grupo incluye variables destacados para los parámetros socio-económicos. Se evaluaron seis criterios restrictivos (tabla 1): a) áreas ambientalmente protegidas, es decir, ecosistemas sensibles siguiendo el Reglamento de la Comisión Europea para la política de biodiversidad natural (NATURA, 2000); b) las zonas en las que está prohibida la construcción de edificios residenciales a tenor de lo indicado por la Ley del Suelo y Ordenación del Territorio de Extremadura; c) la presencia de acuíferos importantes; d) la existencia de superficie de agua (WFD, 2000); e) la vegetación específica y los tipos de uso del suelo y f) los caminos, carreteras y ferrocarriles. Se analizaron cuatro criterios correspondientes a los parámetros de recursos turísticos con los pesos calculados [ratio de consistencia (en adelante CR, de sus siglas en inglés) 0,083 ]: g) el tipo de vegetación $(0,57)$; h) la proximidad de las aguas superficiales $(0,25)$; i) la proximidad de fuentes de agua $(0,12) ; \mathrm{j})$ la visibilidad desde los caminos, carreteras y ferrocarriles $(0,06)$. Se evaluaron cuatro criterios relevantes para los parámetros ambientales con los pesos calculados $(\mathrm{CR}=0,059) ; \mathrm{k})$ la proximidad de ecosistemas sensibles $(0,56)$ (NATURA, 2000); 1) el tipo de uso y cobertura del suelo $(0,25)$ (LESOTEX, 2001); 
m) la pendiente de la superficie terrestre $(0,07)$ y n) la altitud $(0,12)$. Por último, cuatro criterios relevantes para parámetros socio-económicos fueron examinados con los pesos calculados $(\mathrm{CR}=0,091)$; o) la proximidad a las zonas residenciales $(0,13)$; $p$ ) la proximidad a las zonas urbanas $(0,26) ; q)$ el acceso al emplazamiento $(0,56)$ y r) la densidad de la población $(0,05)$ como se establece en el INE (INE, 2011).

TABLA 1

SUB-CRITERIOS DE LOS CRITERIOS DE EXCLUSIÓN

\section{Criterios de exclusión}

Sub-criterios

a) Ecosistema sensible

b) Ordenanza local de construcción

c) Acuíferos relevantes Cuerpos de aguas
superficiales

e) Superficie vegetal y tipos de uso de suelo

f) Carreteras y vías férreas

\section{Contexto del sub-criterio}

Áreas medioambientalmente protegidas por la regulación de la comisión Europea para la naturaleza y la política de biodiversidad, NATURA 2000.

Áreas prohibidas por las legislaciones locales de construcción, donde las edificaciones pueden degradar las áreas ambientales o culturales.

Áreas de manantiales y aguas subterráneas con alto grado de riesgo de contaminación (Gemitzi et al., 2007).

Áreas a una distancia mínima de corrientes de aguas principales o secundarias para prevenir la contaminación de las aguas superficiales siguiendo las obligaciones dispuestas en la directiva europea del Agua (WFD, Directive 2000/60/EC).

Áreas de vegetación densa utilizando él índice normalizado de vegetación diferenciada (NDVI) basado en las imágenes satelitales de Landsat del modelo de elevación digital (DEM).

Áreas contiguas a los límites legales de distancias mínimas a carreteras y vías de férreas. 
TABLA 2

SUB-CRITERIOS BASADOS EN LAS CARACTERÍSTICAS DE LOS RECURSOS

TURÍSTICOS DE LA ZONA DE ESTUDIO DE CASO CON LOS PESOS

CALCULADOS DE LOS FACTORES

Criterios de los recursos turísticos

\begin{tabular}{ccc}
\hline Sub-criterios & Contexto del sub-criterio & $\begin{array}{c}\text { Pesos } \\
\text { calculados }\end{array}$ \\
\hline
\end{tabular}

Áreas que incluyen una evaluación basada

g) Tipo de vegetación

en la singularidad ecológica de la vegetación boscosa y la propagación espacial de estas formaciones naturales basados en el NDVI con el DEM.

Áreas calculadas utilizando funciones de

h) Proximidad a aguas
superficiales distancia euclidiana, la distancia radial desde los recursos de aguas superficiales, lagos y ríos con corrientes de agua continuas.

Áreas calculadas utilizando funciones de

i) Proximidad a cuerpos de agua distancia euclidiana, distancia directa a , 12 cuerpos de agua, manantiales y pozos.

Áreas destinadas a la protección estética

j) Visibilidad desde carreteras y vías férreas utilizando distancias radiales y de visibilidad desde los puntos de acceso al sitio, tales como carreteras (autopistas y carreteras , 06 locales) y los ferrocarriles.

$$
\lambda_{\text {max }}=4,225, \mathrm{CI}=0,075, \mathrm{RI}_{4}=0,9 \mathrm{y} \mathrm{CR}=0,083<0,1
$$


TABLA 3

SUB-CRITERIOS BASADOS EN LAS CARACTERÍSTICAS AMBIENTALES DE LA ZONA DE ESTUDIO DE CASO CON LOS PESOS DE LOS FACTORES

CALCULADOS

\begin{tabular}{|c|c|c|c|}
\hline \multicolumn{4}{|c|}{ Criterios ambientales } \\
\hline & Sub-criterios & Contexto del sub-criterio & $\begin{array}{l}\text { Pesos } \\
\text { calculados }\end{array}$ \\
\hline k) & $\begin{array}{l}\text { Proximidad de } \\
\text { ecosistema sensible }\end{array}$ & $\begin{array}{l}\text { Áreas calculadas utilizando funciones de } \\
\text { distancia euclidiana, distancia radical } \\
\text { desde los ecosistemas sensibles de acuerdo } \\
\text { con el Reglamento de la Comisión Europa } \\
\text { para la Naturaleza y la política de biodiver- } \\
\text { sidad (NATRUA, 2000). }\end{array}$ &, 56 \\
\hline 1) & $\begin{array}{l}\text { Uso de suelo y tipo } \\
\text { de cubierta }\end{array}$ & $\begin{array}{l}\text { Áreas destinadas al desarrollo económico } \\
\text { que cubren diferentes tipos de uso de } \\
\text { suelo (LESOTEX). }\end{array}$ &, 25 \\
\hline $\mathrm{m})$ & $\begin{array}{l}\text { Pendiente superficie } \\
\text { del terreno }\end{array}$ & $\begin{array}{l}\text { Áreas que muestran derivación de atribu- } \\
\text { tos ambientales de procesos paisajísticos } \\
\text { por el flujo de la superficie de terreno, ex- } \\
\text { presado en grados. }\end{array}$ & 07 \\
\hline n) & Elevación & $\begin{array}{l}\text { Áreas que muestran la derivación de los } \\
\text { atributos ambientales de a superficie de te- } \\
\text { rreno por procesos atmosféricos }\end{array}$ & 12 \\
\hline & & \multicolumn{2}{|c|}{$\lambda_{\max }=4,158, \mathrm{CI}=0,053, \mathrm{RI}_{4}=0,9 \mathrm{y} \mathrm{CR}=0,059<0,1$} \\
\hline
\end{tabular}


TABLA 4

SUB-CRITERIOS DE LAS CARACTERÍSTICAS SOCIO-ECONÓMICAS DE LA ZONA DE ESTUDIO DE CASO CON LOS PESOS DE LOS FACTORES CALCULADOS

\begin{tabular}{|c|c|c|c|}
\hline \multicolumn{4}{|c|}{ Criterios socio-económicos } \\
\hline & Sub-criterios & Contexto del sub-criterio & $\begin{array}{c}\text { Pesos } \\
\text { calculados }\end{array}$ \\
\hline o) & $\begin{array}{l}\text { Proximidad a las áreas } \\
\text { residenciales }\end{array}$ & $\begin{array}{l}\text { Áreas calculadas mediante funciones de } \\
\text { distancia euclidiana, distancia radial desde } \\
\text { ciudades y pueblos que representan una } \\
\text { alta concentración de actividades huma- } \\
\text { nas. }\end{array}$ &, 13 \\
\hline p) & $\begin{array}{l}\text { Proximidad a las } \\
\text { zonas urbanas }\end{array}$ & $\begin{array}{l}\text { Áreas calculadas mediante funciones de } \\
\text { distancia euclidiana, distancias directas } \\
\text { desde áreas urbanas basadas en el uso del } \\
\text { terreno y el tipo de cubierta. }\end{array}$ &, 26 \\
\hline q) & Acceso al sitio & $\begin{array}{l}\text { Áreas calculadas mediante funciones de } \\
\text { distancia elucídelas, distancias directas } \\
\text { desde la infraestructura de acceso al sitio, } \\
\text { como carreteras, caminos vecinales y vías } \\
\text { férreas. }\end{array}$ &, 56 \\
\hline r) & Densidad de población & $\begin{array}{l}\text { Áreas que muestre influencia económica } \\
\text { alrededor de las ciudades, pueblos y asen- } \\
\text { tamientos humanos basado en los datos } \\
\text { del INE (instituto nacional de estadística, } \\
\text { 2011) de España. }\end{array}$ &, 05 \\
\hline & & $247, \mathrm{CI}=0,082, \mathrm{RI}_{4}=0,9 \mathrm{y} \mathrm{CR}$ & $, 091<0,1$ \\
\hline
\end{tabular}

La estructura jerárquica del proceso de planificación espacial consta de cuatro niveles: el primer paso muestra el objetivo principal, la pertinencia de la planificación de una segunda residencia; el segundo paso representa criterios que necesitan ser excluidos y evaluados, basados en la primera prueba, y apoyar el objetivo principal; el tercer paso son los sub-criterios de cada criterio; y el último paso muestra los atributos espaciales de cada sub-criterio. El 
proceso se centra en presentar la aplicabilidad del OWA dentro del marco de evaluación multi-criterio más que en explicar o justificar completamente el uso de todos los criterios importantes. La evaluación multi-criterio es un método subjetivo y por tanto sus resultados dependen tanto del propio método como de los criterios seleccionados.

\section{Proceso de asignación de pesos y agregación de criterios}

Según los criterios exhaustivamente seleccionados, los pesos indican la importancia de un criterio relativo a todos los demás criterios y controlan como los criterios se compensan mutuamente en cada grupo de criterios, basándose en la postura de los residentes y la opinión del grupo de expertos. Después de estandarizar todos los criterios para una escala común de 0-255 usando funciones de pertenencia difusa, los pesos de los criterios se repartieron entre todos los criterios de cada grupo. Comenzando con el AHP, se consiguió el peso de importancia relativa utilizando el PCM y se obtuvieron los valores de clasificación para examinar y juzgar la condición actual de los indicadores bajo cada criterio junto con su participación. A continuación, el método de ponderación OWA fue utilizado para el cálculo del índice de idoneidad (Eastman, 2003; Gemitzi et al., 2007). La primera prueba mediante criterios de exclusión del grupo 1 representa el área de estudio en dos categorías de suelos: adecuado (índice de idoneidad 1) e inadecuado (índice de idoneidad 0). La formulación matemática para el área seleccionada, utilizando solamente criterios vinculantes, se aprecia en la Ecuación [1]:

$$
S I=\prod_{k=1}^{l} y_{k}
$$

Donde: SI es el índice de idoneidad total medido de 0 a 1 ; es el criterio de puntuación de la restricción; es el número de criterios vinculantes. Como se muestra en la figura 4, se evaluaron seis criterios vinculantes en el área de estudio. El valor 0, simbolizado con la tonalidad más oscura, representa las áreas que quedan excluidas y no son consideradas, mientras que con el valor 1 , representado con la tonalidad más clara, aparecen aquellos espacios en los que es posible una evaluación adicional. 

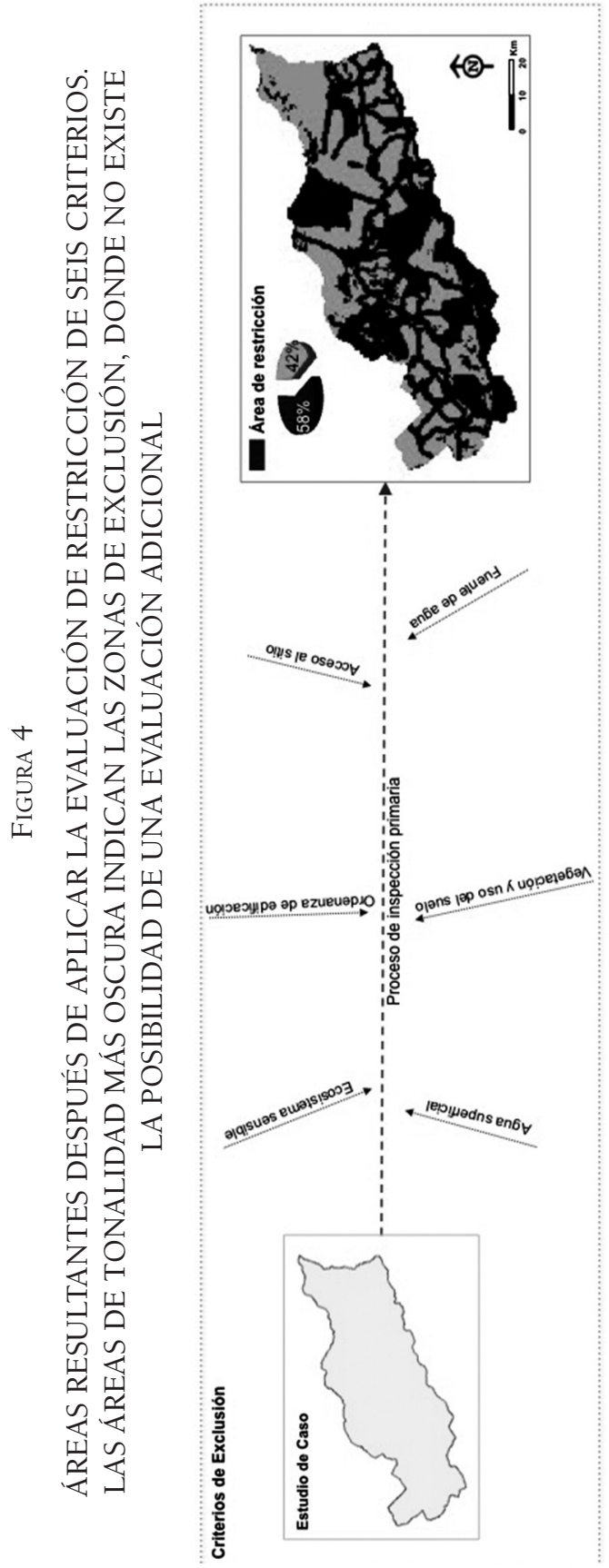

Estudios Geográficos, Vol. LXXVII, 280, pp. 191-216, enero-junio 2016 ISSN: 0014-1496, eISSN: 1988-8546, doi: 10.3989/estgeogr.201607 
El método, el operador OWA (Yager, 1988), es una técnica para criterios de clasificación y abordar la incertidumbre de su interacción, así como agregar múltiples entradas que se encuentran entre los operadores max y min. Este operador tiene como objetivo una agregación no lineal de objetos considerados, siendo diferente de los métodos de agregación multi-atributos existentes (von Winterfeldt \& Edwards, 1986). La principal razón para usar este método en muchas áreas diferentes es su gran flexibilidad a la hora de modelar una gran variedad de agregadores, así como que su naturaleza viene definida por un vector de ponderación y no por un único parámetro (Fernandez-Salido \& Murakami, 2003). Por consiguiente, seleccionando adecuadamente el vector de ponderación, es posible modelar diferentes tipos de relaciones entre criterios agregados. En la implementación espacial, el OWA está definido por una ubicación i-posición (celda ráster) y un conjunto de pesos ordenados $\mathrm{w}=\mathrm{wl}$, w2, .., wn como

$$
w_{j}[0,1] \text { donde } 1,2, \ldots, \text { n y } \sum_{j=1}^{n} w_{j}=1
$$

En cada grupo de criterios, los pesos fueron asignados en función de la importancia que se consideraba que tenía criterio. Teniendo en cuenta el conjunto de valores de los atributos ail, ai2, . . , ain en la ubicación i-posición de una serie de $\mathrm{n}$ mapas de criterios, estos fueron representados por cartografía ráster. La ecuación matemática para la asignación del índice de idoneidad global, aplicando criterios tanto excluyentes y como no excluyentes, es:

$$
S I=\sum_{j=1}^{n} w_{j} x_{i j} \cdot \prod_{k=1}^{l} y_{k}
$$

Donde: SIi es el valor del índice de idoneidad global; $\mathrm{w}_{\mathrm{j}}$ es el peso del factor $\mathrm{j} ; \mathrm{x}_{\mathrm{ij}}$ es la puntuación del criterio del factor $\mathrm{j} ; \mathrm{y}_{\mathrm{k}}$ es la puntuación del criterio de restricción k; es el número de factores; y l es el número de los criterios vinculantes. Esta técnica de agregación multiplica las puntuaciones de los factores por su peso de factor y luego suma los productos para obtener la calificación de idoneidad según lo descrito en la Ecuación [3].

Basándose en la Ecuación [3], se introduce el concepto de orness y la definición de una medida para el mismo que se puede establecer como «orlike» de un determinado operador, utilizando los valores de su función de ponderación. Por lo tanto, la medida puede interpretarse como el modo en que las circunstancias influyen en la toma de decisiones, afectando a las ponderaciones utilizadas en el proceso de agregación (Fernandez-Salido \& Murakami, 2003). La agregación «orlike» persigue el máximo de los objetos ordenados. Este 
concepto coincide perfectamente con la teoría tradicional de toma de decisiones en la que el principio de decisión máximo $(\max )$ expone la decisión más optimista, y el principio de decisión mínimo ( $\mathrm{min}$ ) muestra el contexto de la decisión más pesimista. En esta investigación, se presentan formas analíticas de las funciones de ponderación del operador OWA teniendo cada una de ellas propiedades, tales como pesos basados en el rango y valor constante del orness, independientemente del número agregado de los objetivos (tabla 5). En concreto, se proponen las formas analíticas de las funciones de ponderación OWA que pueden colocarse o posicionarse entre 0,667 (2/3) y 0,75 (3/4) en la escala orness.

Los beneficios de usar estos pesos en lugar de otros esquemas de ponderación son los siguientes: en primer lugar, las formas analíticas de las funciones de ponderación pueden ser utilizadas eficientemente sin resolver complicados programas matemáticos una vez que el grado orness se especifica a priori por aquellos que toman las decisiones; en segundo lugar, combinados con los pesos conocidos del operador OWA (max, min y media), se puede generar un valor deseado de orness independientemente del número de objetivos. Usando las combinaciones convexas de funciones de ponderación predeterminadas, esto puede proporcionar los valores constantes de orness: finalmente, los pesos propuestos para el operador OWA con nivel constante de orness muestran tan solo algunas discrepancias en cuanto a la dispersión con pesos derivados del método de máxima entropía OWA (en adelante MEOWA, de sus siglas en inglés). Además, se aplica a los pesos generados a partir de vectores de ponderación predeterminados con valores constantes de orness.

\section{TABLA 5}

PESOS DEL OWA USADOS PARA CONTROLAR LOS CRITERIOS SUGERIDOS. RESULTADOS Y DISCUSIÓN

\begin{tabular}{lcccc}
\hline \multicolumn{5}{c}{ Ponderaciones ordenados } \\
\hline Ranking & $1^{\circ}$ & $2^{\circ}$ & $3^{\circ}$ & $4^{\circ}$ \\
Orness (2/3) &, 4000 &, 3000 &, 2000 &, 1000 \\
Entropía Máxima (2/3) &, 4210 &, 2770 &, 1820 &, 1200 \\
Orness (3/4) &, 5208 &, 2708 &, 1458 &, 0625 \\
Entropía Máxima (3/4) &, 5260 &, 2680 &, 1370 &, 0690 \\
\hline
\end{tabular}

Estudios Geográficos, Vol. LXXVII, 280, pp. 191-216, enero-junio 2016 ISSN: 0014-1496, eISSN: 1988-8546, doi: 10.3989/estgeogr.201607 
En este trabajo se han presentado varios enfoques recientes para la integración de las construcciones rurales empleando SIG y evaluación multi-criterio (De Vriesa et al., 2012; Jeong et al., 2012; Jeong et al., 2013; Jeong et al., 2014b; Tassinari \& Torreggiani, 2006). En el estudio, sin embargo, además de ofrecer todas las ventajas de las técnicas mencionadas, se ha logrado una contribución importante mediante la aplicación de pesos ordenados, es decir, basados en el rango y el valor constante de orness que es independiente del número de objetivos agregados.

El procedimiento se repitió una vez para cada grupo de factores con los datos de las opiniones de los residentes y del panel de expertos, lo cual da como resultado tres mapas de localización de idoneidad intermedios. En el primero de los mapas, los criterios de los recursos turísticos, ya excluidas las áreas que presentaban limitaciones, fueron calculados y luego agregados tal y como se muestra en la figura 5a. El orden de los pesos asignados (orness y máxima entropía) fueron aplicados a cada criterio en función del tipo de vegetación, la proximidad de las aguas superficiales, la proximidad de los cuerpos de agua y la visibilidad de la edificación desde los caminos, carreteras y vías férreas. En el segundo mapa, los criterios ambientales, ya excluidas las limitaciones, fueron ponderados y luego agregados como se muestra en la figura $5 \mathrm{~b}$. Los pesos ordenados asignados fueron aplicados a cada criterio en función de la proximidad de ecosistemas sensibles, de los usos y coberturas del suelo, de la altitud y de la pendiente de la superficie terrestre. En el tercer y último mapa, los criterios socio-económicos, una vez excluidas la limitaciones, fueron calculados y luego agregados como se muestra en la figura $5 \mathrm{c}$. Los pesos ordenados asignados fueron aplicados a cada criterio teniendo en cuenta la accesibilidad del emplazamiento, la proximidad a las zonas urbanas, la cercanía a las zonas residenciales y la densidad de la población. Aquí, basándose en la encuesta a los residentes y el debate del grupo de expertos, se descubre la postura de los participantes en cuanto a la asignación de criterios específicos: estos dieron un peso más importante a los recursos turísticos entre los criterios; y al tipo de vegetación en los recursos turísticos, a la presencia de ecosistemas sensibles en los criterios ambientales y el acceso al sitio en los criterios socio-económicos, en cuanto a los sub-criterios. 
FIGURA 5

MAPAS INTERMEDIOS DE IDONEIDAD A PARTIR DE TRES CRITERIOS DERIVADOS DEL MÉTODO OWA BASADO EN LOS DATOS DE LA ACTITUD DE LOS RESIDENTES Y DEL GRUPO DE DISCUSIÓN DE EXPERTOS PRESENTADOS

EN LAS TABLAS 2 A 4: MAPA RESULTANTE INTERMEDIO COMPUESTO A

PARTIR DE 2/3 ORNESS Y MÁXIMO ORDEN DE PESOS DE ENTROPÍA

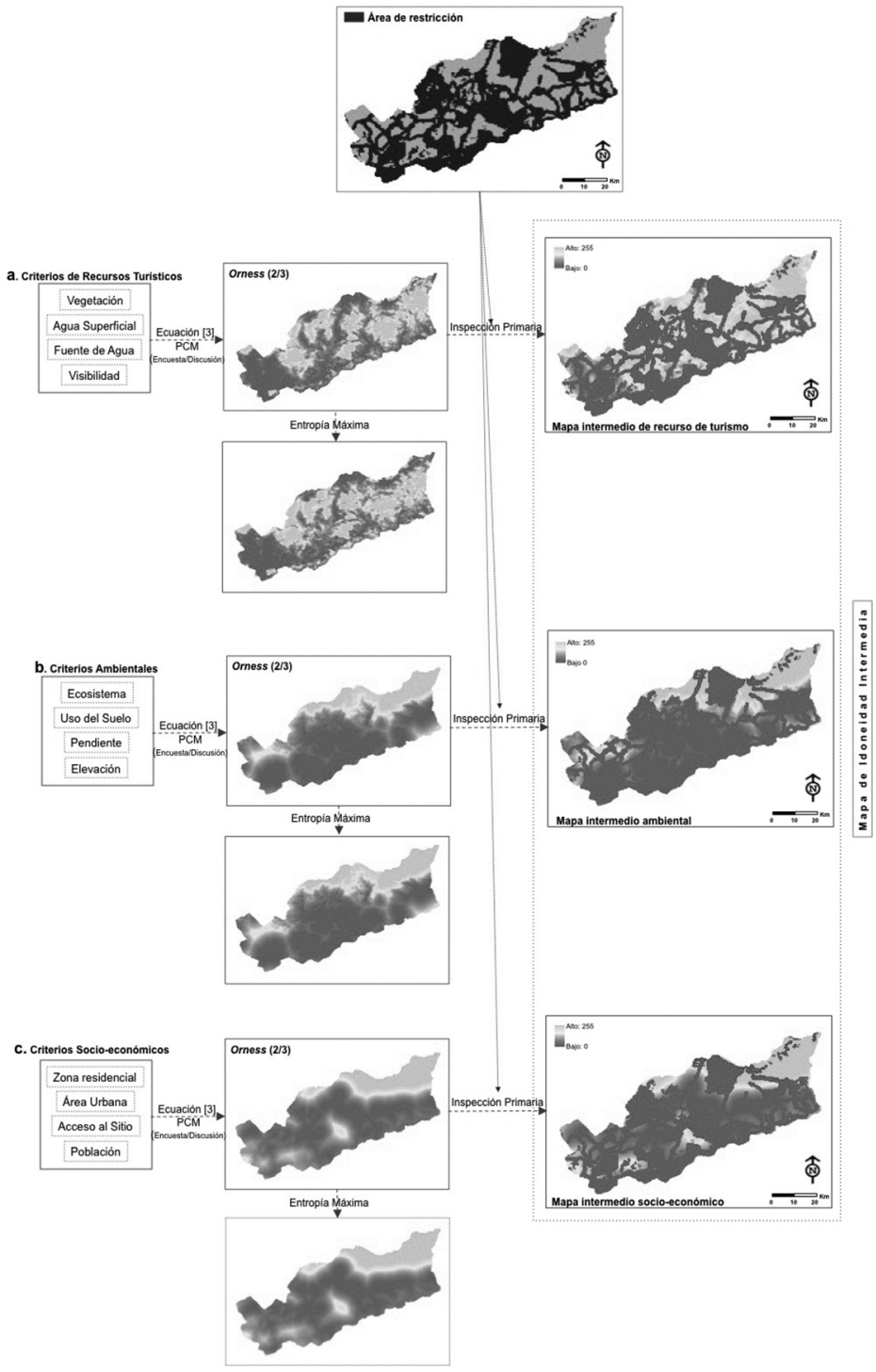

Estudios Geográficos, Vol. LXXVII, 280, pp. 191-216, enero-junio 2016

ISSN: 0014-1496, eISSN: 1988-8546, doi: 10.3989/estgeogr.201607 
Junto con los anteriores resultados intermedios, el mapa final de idoneidad se realizó con el procedimiento de agregación que se muestra en la figura 6. Las limitaciones siempre permanecieron como máscaras Booleanas y no participaron en ningún proceso de asignación de peso. Los mapas de idoneidad intermedios fueron utilizados como funciones de ponderación de $0,667(2 / 3)$ y $0,750(3 / 4)$ en la escala de orness y máxima entropía. Los cuatro pesos ordenados diferentes fueron asignados de igual modo que los resultados intermedios: $1^{\circ}$ puesto: $0,5000,2^{\circ}$ puesto: 0,3330 y $3^{\circ}$ puesto: 0,1670 ( $2 / 3$ de los pesos del orness); $1^{\circ}$ puesto: $0,0514,2^{\circ}$ puesto: $0,3050 \mathrm{y}$ $3^{\circ}$ puesto: 0,1810 ( $2 / 3$ de los pesos de máxima entropía); $1^{\circ}$ puesto: 0,6110 , $2^{\circ}$ puesto: 0,2780 y $3^{\circ}$ puesto: 0,1110 ( $3 / 4$ de los pesos del orness); $1^{\circ}$ puesto: $0,6160,2^{\circ}$ puesto: 0,2680 y $3^{\circ}$ puesto: 0,1160 ( $3 / 4$ de los pesos de máxima entropía). Los pesos ordenados asignados muestran un bajo nivel de riesgo, estando la agregación sesgada hacia el criterio con la puntuación más alta de idoneidad. Los escenarios de agrupamiento posibles fueron ilustrados usando tres criterios importantes, los cuales fueron combinados con 12 sub-criterios y 6 criterios de restricción, tal y como se muestra en la figura 6. Los métodos OWA fueron seleccionados como la forma correcta de solucionar el problema de criterios múltiples para la localización de segundas residencias o viviendas rurales. Por ejemplo, la alternativa (a) aplicada a los 2/3 del orness y la alternativa (b) aplicada a los 3/4 del orness según el orden de los criterios de los recursos turísticos, ambientales y socioeconómicos. Se muestra el porcentaje de las áreas categorizadas: la mejor zona en la alternativa (a) es del 7,00\% con valores entre 200 a 255 del área total; la alternativa (b) asigna las áreas más adecuadas de 7,99\% con valores entre 200 a 255 del área total. En el contexto de los municipios, los resultados muestran cuánto porcentaje de cada área fue asignada a cada territorio (figura 6). Entre los diecinueve municipios de la comarca natural de La Vera, Villanueva de la Vera tiene la mayor tasa de espacio idóneo de ubicación para la evaluación de ambas alternativas. 


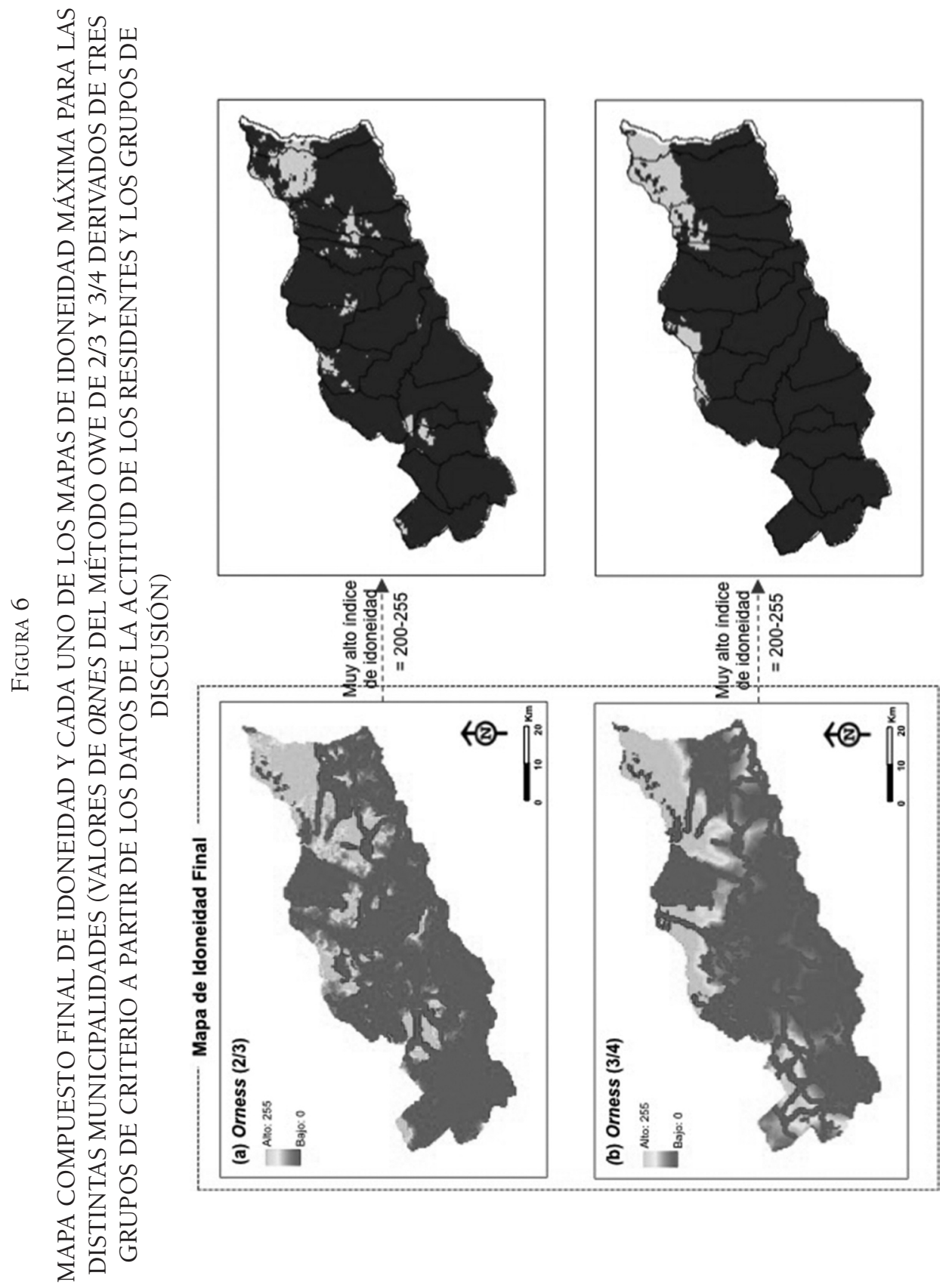

Estudios Geográficos, Vol. LXXVII, 280, pp. 191-216, enero-junio 2016

ISSN: 0014-1496, eISSN: 1988-8546, doi: 10.3989/estgeogr.201607 
De los resultados del proceso de agregación es interesante destacar que la metodología presentada fue capaz de revelar las áreas más adecuadas para la ubicación de segundas residencias rurales dentro de su paisaje, pudiendo conducir, de tal modo, hacia el desarrollo sostenible del turismo, así como proporcionar una clasificación inicial de las áreas más adecuadas. Por lo tanto, es bastante simple explorar diferentes escenarios de ubicación, ya se desee un enfoque más vinculado al ecoturismo, o se apueste por una alternativa ambientalmente más sensible. Considerando que el área de estudio es de 888 $\mathrm{km} 2$, y que los emplazamientos adecuados deben estar comprendidos dentro ésta, se empleará una restricción de agregación solo si hay, al menos, una segunda residencia rural que vaya a ofrecer su servicio en todo el área de estudio. Esto debe ser considerado en una cantidad fija para las mejores ubicaciones, las cuales pertenecen a la quinta categoría de idoneidad (200 a 255), equivalente a la zona requerida. Con respecto a la encuesta, los entrevistadores mostraron el mapa de idoneidad final en base a su selección a los encuestados y les preguntaron cómo afectaría este a su postura inicial acerca de la determinación de las localizaciones idóneas. A tenor de esto, 36 de las 41 respuestas confirmaron su cambio de postura en cuanto a la integración de las segundas residencias tras responder a la encuesta.

Cabe mencionar aquí, que el trabajo presentado no ofrece una decisión final para la localización de segundas residencias rurales, sino un proceso de toma de decisiones basado en un enfoque metodológico para su ubicación. La selección de criterios se limitaba a datos básicos que están actualmente disponibles y que proceden de diferentes fuentes, por consiguiente, se reconoce que se han omitido algunos de los factores claves que potencialmente podrían producir diferentes alternativas de decisión. Sin embargo, el proceso de ubicación con la metodología propuesta descrita en el presente estudio, podría ser muy útil para múltiples participantes, especialmente para la postura tomada por los residentes, en relación con el emplazamiento de las actividades humanas vinculadas con planificación de segundas residencias rurales.

\section{CONCLUSIONES}

En este estudio, se utilizó el enfoque AHP/OWA en combinación con las capacidades analíticas de los SIG. Los resultados de la investigación muestran la efectividad de este método a la hora de determinar los lugares idóneos para integrar segundas residencias rurales, basado en el entendimiento de las limi- 
taciones regionales existentes en la comarca natural de La Vera. En el proceso de cálculo computacional se examinaron seis criterios excluyentes y doce no excluyentes, clasificados en cuatro grupos basados en los resultados de la encuesta llevada a cabo entre los residentes locales y el análisis del grupo de expertos. En la encuesta, los entrevistados mostraron su preferencia de criterios y sub-criterios como sigue: el criterio del recurso turístico fue el más importante; mientras que el tipo de vegetación, la sensibilidad del ecosistema y el acceso al lugar fueron, respectivamente, el sub-criterio más importante en los criterios del recurso turístico, ambiental y socio-económico. Se elaboraron tres mapas de idoneidad intermedia (recurso turístico, ambiental y socio-económico) con los resultados anteriores para, a continuación, combinarlos y crear un mapa de idoneidad compuesto. El AHP ofrecía un proceso de asignación de pesos bastante objetivo. Además, el uso del segundo grupo de pesos, pesos ordenados, proporcionaba gran flexibilidad en el procedimiento de agregación, ofreciendo pesos basados en el rango y un valor constante de orness y máxima entropía OWA, independientemente del número de objetivos agregados.

El principal objetivo de estos resultados preliminares es la implementación concebida de esta metodología flexible, en lugar de considerar todos los parámetros y criterios de evaluación posibles. Asimismo, los resultados de este estudio demuestran que el objetivo del enfoque no es encontrar una única solución óptima, sino mostrar otras fortalezas asociadas con la flexibilidad de la ponderación del enfoque de la función OWA. Otros puntos fuertes de este enfoque incluyen la capacidad de integrar conjuntos de datos heterogéneos como criterios cuantitativos y cualitativos mediante el conocimiento mostrado por los residentes del área de estudio y un grupo de expertos. Su adaptabilidad es muy importante para ponderar criterios específicos en diferentes áreas de estudios o la consideración de diferentes problemas, así como para implementar una única o un grupo de decisiones. Además, la flexibilidad que ofrece la función es básica para cambiar el nivel de importancia de los criterios, presentando la libertad de desarrollar varios escenarios de modelización para niveles aceptables de riesgos en la decisión. En particular, en este estudio, se demuestra que el $87,8 \%$ de los residentes locales han cambiado su opinión en cuanto a la selección la ubicación de una segunda residencia rural, después de revisar el mapa de idoneidad final en el que aparece reflejado su propio proceso de ponderación. 


\section{BIBLIOGRAFÍA}

Allen, L. R., Hafer, H. R., Long, P. T. y Perdue, R. R. (1993): “Rural residents' attitudes toward recreation and tourism development", Journal of Travel Research, 31/4, pp. 27-33.

Andereck, K. L., Valentine, K. M., Knopf, R. C. y Vogt, C. A. (2005): "Residents' perceptions of community tourism impacts", Annals of Tourism Research, 32/4, pp. 1056-1076.

Bell, S. (1995): Elements of visual design in the landscape, London, E\&FN Spon.

Blaschke, T. (2006): "The role of the spatial dimension within the framework of sustainable landscapes and natural capital", Landscape and Urban Planning, 75, pp. 198-226.

Böhme, K. y Schön, P. (2006): "From Leipzig to Leipzig: territorial research delivers evidence for the new territorial agenda of the European Union", disP, 165/2, pp. 61-70.

Byrd, E. T., Cardenas, D. A. y Dregalla, S. E. (2009): "Differences in stakeholder attitudes of tourism development and the natural environment", e-Review of Tourism Research, 7/2, pp. 39-51.

Carver, S. (1999): "Developing Web-based GIS/MCE: improving access to data and spatial decision support tools", en J. C. Thill (ed.), Spatial Multicriteria Decision making and Analysis, Aldershot, England, Ashgate, pp. 49-75.

Council of the European Union (2001): "Council resolution on architectural quality in urban and rural environments (2001/C 73/04)", Official Journal of the European Communities, C73, 6/3/2001.

De Vriesa, S., de Grootb, M. y Boersb, J. (2012): "Eyesores in sight: quantifying the impact of man-made elements on the scenic beauty of Dutch landscapes", Landscape and Urban Planning, 105, pp. 118-127.

Drumm, A. (1998): "New approaches to community-based ecotourism management. Learning from Ecuador", en K. Lindberg, M. E. Wood y D. E. Hawkins (eds.), Ecotourism: a guide for planners and managers, Vermont, The Ecotourism Society, pp. 197-213.

Eagles, P. F. J., McCool, S. F. y Haynes, C. F. (2002): Sustainable tourism in protected areas: guidelines for planning and management, Gland, International Union for the Conservation of Nature.

Eastman, J. R. (2003): IDRISI Kilimanjaro: guide to GIS and image processing, Worcester, Clark Laboratories, Clark University.

Fernandez-Salido, J. M. y Murakami, S. (2003): "Extending Yager's orness concept for the OWA aggregators to other mean operators", Fuzzy Sets and Systems, 139, pp. 515-542.

Fuller, R. y Majlender, P. (2001): "An analytic approach for obtaining maximal entropy OWA operator weights", Fuzzy Sets and Systems, 124, pp. 53-57.

Gallent, N. y Tewdwr-Jones, M. (2000): Rural second homes in Europe - examining housing supply and planning control, Aldershot, England, Ashgate.

García Moruno, L., Hernández Blanco, J., Ayuga Téllez, F. y García Navarro, J. (1998):

Estudios Geográficos, Vol. LXXVII, 280, pp. 191-216, enero-junio 2016

ISSN: 0014-1496, eISSN: 1988-8546, doi: 10.3989/estgeogr.201607 
"Localizacion de las construcciones en el ámbito rural ante la protección del paisaje", Informes de la Construcción, 50/458, pp. 61-70.

Gemitzi, A., Petalas, C., Tsihrintzis, V. y Pisinaras, V. (2007): “Assessment of groundwater vulnerability to pollution: a combination of GIS, fuzzy logic and decision making techniques", Environmental Geology, 51, pp. 797-811.

Guevara, J. R. Q. (1996): "Learning through participatory action research for community ecotourism planning", Convergence, 29/3, pp. 24-40.

Hermann, S. y Osinski, E. (1999): "Planning sustainable land use in rural areas at different spatial levels using GIS and modelling tools", Landscape and Urban Planning, 46, pp. 93-101.

INE (2011): Instituto Nacional de Estadística, http://www.ine.es/jaxi/menu.do?type= pcaxis\&path=\%2Ft 20\%2Fe260\%2Fa2011\%2F\&file=pcaxis\&N=\&L=0 (Fecha de consulta: 16/04/2014).

Jaraíz, F. J., Mora, J., Gutiérrez, J. A. y Jeong, J. S. (2013): "Comparison of regional planning strategies: countywide general plans in USA and territorial plans in Spain", Land Use Policy, 30/1, pp. 758-773.

Jeong, J. S., García-Moruno, L. y Hernández-Blanco, J. (2012): "Integrating buildings into a rural landscape using a multi-criteria spatial decision analysis in GIS-enabled web environment", Biosystems Engineering, 112/2, pp. 82-92.

Jeong, J. S., García-Moruno, L. y Hernández-Blanco, J. (2013): “A site planning approach for rural buildings into a landscape using a spatial multi-criteria decision analysis methodology", Land Use Policy, 32, pp. 108-118.

Jeong, J. S., García-Moruno, L. y Hernández-Blanco, J. (2014a): "Un modelo WEB para la asistencia en la toma de decisiones en la integración de las construcciones rurales mediante planificación espacial multi-criterio", Informes de la Construcción, 66/533, e004. doi: http://dx.doi.org/10.3989/ic.13.001.

Jeong, J. S., Hernández-Blanco, J. y García-Moruno, L. (2014b): “Approaches to validating a mutual participatory web-planning interface in rural Extremadura (Spain)", Land Use Policy, 39, pp. 211-223.

Jeong, J. S., Montero-Parejo, M. J., Hernández-Blanco, J. y García-Moruno, L. (2015): "The visual evaluation of rural areas: a methodological approach for the spatial planning and color design of scattered second homes with an example in Hervás, Western Spain", Land Use Policy, 46, pp. 330-340.

Lai, P. H. y Nepal, S. K. (2006): "Local perspectives of ecotourism development in Tawushan Nature Reserve, Taiwan", Tourism Management, 27/6, pp. 1117-1129.

LESOTEX (2001): Guía práctica de aplicación de la Ley 15/2001 del suelo y ordenación territorial de Extremadura, http://sitex.juntaex.es/sias/Documentacion/guia_prac tica.pdf (Fecha de consulta: 6/09/2014).

Malczewski, J. (1999): GIS and multicriteria decision analysis, New York, John Wiley $\&$ Sons.

Malczewski, J. (2004): "GIS-based land-use suitability analysis: a critical overview", Progress in Planning, 67, pp. 3-65. 
Morales Yago, F. J. (2013): "El impacto de la actividad turistica sobre el paisaje de La Manga del Mar Menor (Murcia)", Estudios Geograficos, 74/275, pp. 523-556.

NATURA (2000): European commission environment for nature E biodiversity policy, http://ec.europa.eu/environment/nature/natura2000/index_en.htm (Fecha de consulta: $23 / 07 / 2014$ ).

Page, S. y Dowling, R. K. (2002): Ecotourism, Harlow, Pearson Education.

Ross, S. y Wall, G. (1999): "Ecotourism: towards congruence between theory and practice", Tourism Management, 20/1, pp. 123-132.

Roy, B. (1996): Multicriteria methodology for decision aiding, Dordrecht, Kluwer Academic Publishers.

Saaty, T. L. (1977): "A scaling method for priorities in hierarchical structures", Journal of Mathematical Psychology, 15, pp. 234-281.

Tandy, C. (1979): Industria y paisaje, Madrid, Ed Leonard Hill Books.

Tassinari, P. y Torreggiani, D. (2006): "Location planning: a methodological approach for agro-industrial buildings in rural territory", Transactions of the ASAE, 49/2, pp. 505-516.

Tewdwr-Jones, M., Gallent, N. y Mace, A. (2002): Second homes and holiday homes and the land use planning system, Cardiff, Welsh Assembly.

Teye, V., Sirakaya, E. y Sönmez, S. (2002): "Residents' attitudes toward tourism development", Annals of Tourism Research, 29/3, pp. 668-688.

Vepsäläinen, M. y Pitkänen, K. (2010): "Second home countryside. Representations of the rural in Finnish popular discourses", Journal of Rural Studies, 26, pp. 194-204.

Vincent, V. C. y Thompson, W. (2002): "Assessing community support and sustainability for ecotourism development”, Journal of Travel Research, 41/2, pp. 153-160.

von Winterfeldt, D. y Edwards, W. (1986): Decision analysis and behavioral research, Cambridge, Cambridge University Press.

Weaver, D. B. (2001): Ecotourism, Milton, Qld, John Wiley \& Sons.

Weaver, D. B. y Lawton, L. J. (2007): "Twenty years on: the state of contemporary ecotourism research", Tourism Management, 28/5, pp. 1168-1179.

WFD (2000): Directive 2000/60/EC of the European Parliament and of the Council of 23 October 2000 establishing a framework for Community action in the field of water policy, http://eur-lex.europa.eu/LexUriServ/LexUriServ.do?uri=CELEX32000L0060: EN:NOT (Fecha de consulta: 17/10/2014).

Yager, R. R. (1988): “On ordered weighted averaging aggregation operators in multicriteria decision making", IEEE Transactions on Systems, Man, and Cybernetics, 8, pp. 183-190.

Zhang H. y Lei, S. L. (2012): "A structural model of residents' intention to participate in ecotourism: the case of a wetland community", Tourism Management, 33, pp. 916-925.

Fecha de recepción: 27 de noviembre de 2014. Fecha de aceptación: 23 de septiembre de 2015. 


\section{RESUMEN}

Se presenta un método de análisis operacional para apoyar la decisión para la implantación sostenible de segundas residencias rurales en un espacio dedicado al ecoturismo: la comarca de La Vera (Extremadura). En este estudio se emplean procesos jerárquicos analíticos (AHP) y promedios ponderados ordenados (OWA) como técnicas de modelado con múltiples atributos junto a las capacidades que ofrecen los sistemas de información geográfica (SIG). Se analizan seis criterios seleccionados de forma restrictiva y otros doce de forma no restrictiva, basados en todas las características posibles de las políticas de planeamiento existentes junto con dos procedimientos analíticos: encuesta a los residentes locales y grupos de discusión de expertos. A través de operadores del OWA con valor constante del orness y máxima entropía, los lugares óptimos para la localización de las segundas residencias rurales se representan en el área de estudio para evaluar las implicaciones ambientales actuales y predecir las mejoras futuras.

PAlAbRAS ClAVE: segunda vivienda rural; AHP/OWA; evaluación multi-criterio; toma de decisiones; postura residentes.

\section{ABSTRACT}

This paper presents an operational analysis method to support siting decisions for sustainable rural second homes in an ecotourism site, La Vera (Extremadura). In this study, the analytical hierarchy process (AHP) and ordered weighted averaging (OWA) approach of multi-attribute modeling are used in combination with geographic information system (GIS) capabilities. Accordingly, this work explores six exclusionary and twelve non-exclusionary criteria selected based on all possible characteristics of the existing planning policies together with two analytical procedures. Local residents' field survey and experts' group discussion are conducted to reach a consensus criteria weight and particularly to demonstrate their attitude. Then, with the aid of OWA operators with constant value of orness and maximum entropy, the optimal sites for a rural second home are represented in the study area to evaluate the present environment and to predict future improvements.

KEY WORDS: rural second home; AHP/OWA; multi-criteria evaluation; decision-making; residents attitude. 